\title{
Costos de producción en explotaciones porcinas de ciclo completo en el Municipio Mara, estado Zulia, Venezuela*
}

\author{
Rodríguez Medina, Guillermo** \\ Rodríguez Castro, Belkis*** \\ Villasmil, Amaloa Karina****
}

\section{Resumen}

El presente artículo tiene como objetivo analizar los costos de producción en las explotaciones porcinas de ciclo completo en el municipio Mara, estado Zulia, Venezuela, debido a que es un factor clave para la competitividad del sector, además de ser de gran importancia para la soberanía y seguridad alimentaria de la población. El estudio se sustentó en los enfoques o teorías sobre costos de producción y el conocimiento de las fases de producción del sector, expuestos por los autores Gadd (2005), Guzmán (2007), Sábata (2008), Hansen y Mowen (2007), Horngren et al (2007), entre otros. El tipo de investigación fue descriptiva, de diseño no experimental, transeccional y de campo, aplicando un cuestionario a propietarios o administradores de las explotaciones objeto de estudio. Los resultados indican que sólo una granja determina su costo de producción, y todas ellas reconocen el alimento como el recurso de mayor impacto dentro de los costos directos, pero no conocen la ponderación de los elementos que los integran ni los costos indirectos dentro de la estructura de costo; se concluye que el sector estudiado no realiza una buena gestión de sus costos de producción, lo que restringe el éxito en dichas granjas; por lo que se considera deben asumir recomendaciones para potenciarlo.

Palabras clave: Costos de producción, costos directos e indirectos, fases de producción, explotaciones porcinas de ciclo completo, municipio Mara- Edo. Zulia.

Recibido: 10-02-12. Aceptado: 31-10-12

* Este artículo es resultado del proyecto de investigación: Costos de producción en las explotaciones porcinas de ciclo completo, financiado por el Consejo de Desarrollo Científico y Humanístico (CONDES) de la Universidad del Zulia (LUZ).

** Doctor en Ciencias. Mención Gerencia, Profesor Titular Emeritus de LUZ, Investigador del Centro de Estudios de la Empresa (CEE). Acreditado en el PEII Nivel C. e-mail: rodrigue guillermo@gmail.com y grodriguezm@luz.edu.ve

*** Magister en Gerencia de Empresas. Mención Gerencia Financiera (LUZ), Profesora de la Universidad Rafael Belloso Chacín (URBE), co-investigadora auxiliar del CEE de LUZ. Acreditada en el PEII Nivel A, e-mail: rodriguezcbelkis@gmail.com

**** Magíster en Gerencia de Empresas. Mención Gerencia de Operaciones. Ingeniera Química. Asistente de investigación del CEE de LUZ. Acreditada en el PEII Nivel A. e-mail amaloa villasmil@yahoo.com 
Costos de producción en explotaciones porcinas de ciclo completo en el Municipio Mara...

Rodríguez Medina, Guillermo; Rodríguez Castro, Belkis y Villasmil, Amaloa Karina

\title{
Production Costs in Complete Cycle Pig Holdings in the Mara Municipality, State of Zulia, Venezuela
}

\begin{abstract}
The objective of this articles it to analyze production costs on complete cycle pig farms in the Mara Municipality, State of Zulia, Venezuela, since this is a key factor for competitiveness in the sector, as well as having a great importance for the food sovereignty and security of the population. The study was supported on the approaches or theories about production costs and knowledge about production phases in the sector, expressed by the authors Gadd (2005), Guzmán(2007), Sábata (2008), Hansen and Mowen (2007) and Horngren et al. (2007), among others. Research was of the descriptive type, with a non-experimental, trans-sectional, field design, applying a questionnaire to owners or administrators of the pig farms under study. Results indicate that only one farm determines its production costs, and all of them recognize feed as the resource with the greatest impact on direct costs; however, they do not know the weighting of the direct cost elements nor the indirect costs in the cost structure. Conclusions are that the sector under study does not have good production cost management, thereby restricting success on these farms. Recommendations should be considered for empowering success.
\end{abstract}

Key words: Production costs, direct and indirect costs, production phases, complete cycle pig farms, Mara municipality, State of Zulia.

\section{Introducción}

La porcicultura en Venezuela en los últimos años ha sufrido grandes transformaciones y ha dejado de ser una actividad casuística o tradicional para convertirse en una actividad económica lucrativa, por tanto hay que velar por la eficiencia productiva, en búsqueda de los mejores beneficios para los productores, trabajadores y para la sociedad en general; de manera que se garantice a esta la seguridad y soberanía alimentaria en este segmento.

Para la consecución de la eficiencia productiva se hace necesario que dichas explotaciones pongan en práctica herramientas gerenciales, principalmente en factores críticos de éxito; entre ellos los costos de producción, la reproducción, la genética, la nutrición, la sanidad, el mane- jo, entre otros; esto implica llevar un registro sistemático de sus operaciones, hacer los análisis respectivos, así como su evaluación; aspectos que permitirán mejorar la toma de decisiones, en cuanto a la reducción de los costos, al incremento de la productividad y a la calidad de la carne. Sin embargo, estos factores pueden acarrear una serie de inconvenientes que le impiden al sector (que ha pasado a ser un negocio apetecido) mejorar su redituabilidad; pudiendo mencionarse entre ellos el desconocimiento pormenorizado de los elementos de la estructura de costos, sus procesos y actividades de forma integral, aunado a los pocos estudios desarrollados en el sector que sirvan de orientación a los productores.

En tal sentido, el presente trabajo tuvo como objetivo analizar los costos de 
producción en las explotaciones porcinas de ciclo completo en el municipio Mara del estado Zulia, Venezuela. Se abordaron tópicos sobre los diferentes tipos de granjas porcinas, las fases del proceso de producción, si estas se concentran en uno o varios sitios, la importancia de la inseminación artificial, y por ende los costos de producción, considerando los costos directos e indirectos, los procesos, las actividades, los recursos y el sistema de costeo.

La investigación fue de tipo descriptivo, debido a que "tiene como objetivo lograr la precisión y caracterización del evento de estudio dentro de un contexto particular" (Hurtado, 2010:413), de diseño no experimental, dado que no se manipularon intencionalmente las variables, pues, se observaron los fenómenos tal como se dieron en la realidad para su descripción; transeccional porque los datos fueron recopilados en un tiempo único, de campo por cuanto la información primaria se obtuvo directamente de las granjas que fungieron como unidades de análisis.

Merece atención especial, la población estudiada, debido a que no existe un directorio actualizado que contenga el universo de las granjas porcinas en el municipio Mara del estado Zulia, Venezuela, se tuvo la oportunidad de visitar varias instancias como el Ministerio del Poder Popular de Agricultura y Tierra (MPPAT), el Instituto Nacional de Sanidad Agrícola Integral (INSAI) adscrito al MPPAT, del estado Zulia, y del municipio Mara, al Colegio de Médicos Veterinario y sólo se pudo conseguir la data del censo agrícola realizado en el año 2008, el cual está desactualizado.
Por otro lado, se invitó a un conversatorio con la Directiva de la Federación Venezolana de Porcicultura (FeporcinaZulia), en el cual asistió su Presidente y su Vicepresidente, quienes también manifestaron que no existe un directorio de reciente data de este sector, sin embargo el Vice-presidente de la Federación quien es productor en el sector porcino brinda su colaboración conjuntamente con otros granjeros conocedores de la zona. Ambos sirvieron de guía en el desarrollo del trabajo de investigación, y de esta manera se pudo llevar a cabo.

Esta situación particular permitió asumir un muestreo no probabilístico cuya selección fue por situaciones de conveniencia y facilidad de acceso a la información, denominado opinático y bola de nieve (Parra, 2006:25-26), consistiendo el primero en hacer un esfuerzo deliberado de obtener muestras representativas y el segundo en que una vez se encuentre a cierto productor, este conduce a otros, y así sucesivamente hasta que se entrevistó a diecinueve (19) granjeros, considerándose una muestra representativa del sector objeto de estudio

Los datos se recopilaron aplicando un cuestionario estructurado mediante entrevistas a los productores o administradores; la data recabada se procesó a través del paquete estadístico SPSS versión 17, insumo fundamental para la interpretación y análisis de los resultados.

\section{Explotaciones porcinas de ciclo completo: Consideraciones generales}

En esta sección se abordan algunas consideraciones generales, los dife- 
Costos de producción en explotaciones porcinas de ciclo completo en el Municipio Mara... Rodríguez Medina, Guillermo; Rodríguez Castro, Belkis y Villasmil, Amaloa Karina

rentes tipos de granjas, el proceso de producción, su concentración en uno o varios sitios, así como, la importancia de la inseminación artificial.

Debido a la creciente demanda mundial de alimentos para el consumo humano, la carne de cerdo se constituye en una buena alternativa, ya que posee una valiosa fuente de proteínas, energías, vitaminas y minerales. Tanto es así que, según Araque (2009), la porcicultura representa la principal actividad pecuaria y fuente de proteína animal a nivel mundial, equivalente al $42 \%$ de la producción total de carne del mundo.

Actualmente la explotación porcina se considera como un sistema abierto que interactúa con el entorno, pues, las organizaciones no son ni autosuficientes ni cerradas, necesitan alimentarse de elementos externos para llevar a cabo sus operaciones, tal como la materia prima y la fuerza de trabajo, entre otros. Aunado a ello, para que dicha explotación sea competitiva es necesario tomar en cuenta lo recomendado por Maqueda (2004), citado por Utrera (2006), donde indica que la producción porcina debe apoyarse encuatro elementos interrelacionados, como son: "la genética, la nutrición, el manejo y la sanidad". Esto está en concordancia con lo que indica Moreno (2000:2), quien señala que "la cría de cerdo es altamente productiva siempre y cuando los que se dedican a ella adopten los sistemas o métodos indicados más eficientes, para sacar mejor provecho a su explotación".

Por otra parte, el ganado porcino tiene características muy peculiares que hacen atractiva la actividad para su explotación, entre estos el corto período desde su gestación hasta la fase de engorde para el consumo humano y su alta proliferación, pues, de acuerdo a Guzmán (2007:13).

"Un productor de cerdos, hoy en día, empleando razas adecuadas, con una buena alimentación y prácticas sanitarias eficaces desde el nacimiento hasta los siete meses puede lograr un peso de 100 kgs en pie, con una conversión promedio de alimentos de 3,5 kgs por kilogramo de carne, lo que significa que se requiere de cerca de $350 \mathrm{kgs}$ de alimento por cerdo para lograr una buena rentabilidad".

Esta última situación es lo que Gadd (2005) denomina tasa de conversión del pienso (FCR, Food conversión ratio), la cual se determina en función ya sea del peso vivo, del peso en canal o de la carne magra.

Dentro de éste contexto, las granjas porcinas en el municipio Mara del estado Zulia, Venezuela, no se alinean con los parámetros establecidos para explotar dicha actividad de una manera más lucrativa, tanto desde el punto de vista económico como social; los productores de dicho municipio, con pocas excepciones, se dedican a estas explotaciones por la convicción de su rentabilidad, pero sin poseer conocimientos necesarios para la planificación y el control de los factores clave antes mencionados, ni siquiera con el de genética, que de acuerdo a la serie de Manuales para educación agropecuaria (2006:19) se indica que "la explotación porcina comienza por la obtención de buenos reproductores. Esto se logra al aplicar conocimientos especializados en la selección y el cruzamiento, así como en el apareamiento consanguíneo y la inse- 
minación artificial, para mejorar las razas y aumentar su productividad".

Para que la explotación porcina sea más redituable y se propenda la seguridad y soberanía alimentaria del municipio, es necesario hacer uso más eficiente de los recursos utilizados: humanos, físicos y financieros, así como llevar a cabo el proceso administrativo de una manera sistematizada, que de acuerdo a Amarú (2009), Da Silva (2003) y Stoner y Freeman (1995), no es más que planear, organizar, dirigir y controlar. Esta situación también es válida para gestionar los costos de producción.

\subsection{Tipos de granjas o explotaciones}

Existen diversas formas para llevar a cabo la explotación porcina, las cuales de acuerdo a lo indicado tanto por Sábata (2008) como por el Centro Nacional de producción más limpio de Honduras CNP+LH, (2009), las clasifican en tres tipos:

Granjas de cría: Son aquellas que se dedican a producir cerdo desde su nacimiento hasta el destete, con un promedio de 115 días de gestación, 21 días de lactancia y 7 días de destete, con un peso promedio de 15 kilos, para ser vendidos, teniendo como norte obtener el máximo número de lechones destetados por cerda al año. Estas granjas por lo general manejan reproductores, hembras de reemplazo, hembras en gestación, hembras en lactancia, hembras vacías, hembras de descartes y lechones destetados.

Granjas de engorde: Son aquellas que se dedican a engordar cerdos machos y hembras, con una buena calidad de la carne, para luego ser sacrificados o vendidos, de manera que se busca que el producto final se adapte a las exigencias del mercado. Estas granjas compran lechones destetados, con un peso promedio de 15 kilos y los engordan hasta un peso promedio de 100 kilos, en un período que va desde el levante (60 días), desarrollo y crecimiento (30 días) hasta el engorde (45 días).

Granjas de ciclo completo o cerrado: Son las granjas que realizan las actividades de cría y engorde, pues, éstas explotaciones tienen sus propios reproductores (hembras y machos), ya sean comprados o criados, los lechones nacen en la misma granja, donde son criados y engordado hasta su sacrificio o venta.

\subsection{Descripción del proceso de producción de explotaciones porcinas de ciclo completo o cerrado}

En este tipo de explotación, de acuerdo al Centro Nacional de Producción más limpio de Honduras (2009), Sábata (2008) y Moreno (2000), el proceso de producción tiene una serie de fases o etapas que se describen a continuación:

Fase de reproducción: en esta etapa cuando a la cerda reproductora se le detecta el celo, que puede ser primeriza o multípara (varios partos), se realiza la cubrición, el servicio o el apareamiento, ya sea por monta natural o inseminación artificial (la importancia de ésta última se desarrolla más adelante), en corrales conocidos como salas de monta, y se recomienda practicarla por lo menos dos veces para garantizar que la cerda reproductora quede preñada; posteriormente, aproximadamente a los 21días después, se realiza un control por el método de re- 
Costos de producción en explotaciones porcinas de ciclo completo en el Municipio Mara... Rodríguez Medina, Guillermo; Rodríguez Castro, Belkis y Villasmil, Amaloa Karina

torno, o se espera hasta el día 30 para realizar el diagnóstico por ecosonografía a través de la detección de vesículas en el útero, para determinar sí efectivamente resulta positiva y que no haya repetición del celo, si realmente resulta preñada se pasa a la siguiente etapa.

Fase de gestación: después de confirmada el estado de preñez, se pasa a la fase de gestación, en jaulas individuales, donde el peso vivo de una cerda al comienzo de la gestación fluctúa, de acuerdo a Babot et al (2003), citado por Sábata (2008:66), entre los 130 kgs para una cerda joven hasta unos $200 \mathrm{kgs}$ para una cerda multípara o de varios partos, y se le dará el cuidado correspondiente, tanto desde el punto de la alimentación como de las condiciones de alojamiento, con la finalidad de que llegue a la fase del parto sin ningún tipo de inconveniente y evitar así un aborto. En esta fase la cerda dura unos 115 días aproximadamente y una semana antes del parto pasa a la fase de maternidad, también conocida como sala de espera o preparto.

Fase de parto y maternidad: en esta fase, de acuerdo a Whittemore (1998) citado por Sábata (2008), se presentan tres momentos: 1) el de preparatoria, 2) el de expulsión de los fetos y 3) el de la placenta. En la sección de preparatoria o de espera, es decir, una semana antes del parto la cerda presenta una serie de modificaciones conductuales, anatómicas y fisiológicas, debe ser desparasitada y bañada para mantenerla limpia al momento que se presente dicho parto.

En la sección de expulsión cuando el parto es normal, el tiempo transcurrido entre un lechón y otro, es de aproximadamente de 15 minutos y la duración del parto es de aproximadamente de dos a cuatro horas, dependiendo, entre otras cosas del tamaño de la camada o sea el número de lechones nacidos en el parto, que podría ser de ocho (8) a catorce (14); por último, la sección de la placenta, que se caracteriza por la expulsión de las membranas fetales debido a que las contracciones uterinas continúan, deben ser retiradas, para luego proceder a la limpieza de los lechones, que normalmente tienen un peso promedio entre 1 y 1,5 kilogramos (kgs).

Esta situación obliga a los productores de cerdos a por lo menos tener como mínimo un registro de la fecha probable de parto y un programa de asistencia cuando ocurra el mismo.

Después del parto comienza el período de maternidad o lactación, donde la producción de leche en la cerda depende de factores inherentes a la reproductora, como la genética y a factores externos como factores ambientales, de alimentación y sanitarios, por eso ésta fase requiere de una atención más personalizada.

El período de lactancia es de aproximadamente de 21-28 días, cuando se destetan, es recomendable que a los pocos días de nacidos se le corte el rabo y los colmillos para mantener un buen estado sanitario. La alimentación del lechón es la leche materna y una semana antes del destete se le debe dar el alimento Preiniciador para acostumbrarlos a la alimentación balanceada.

El objetivo fundamental de toda granja porcina es obtener el mayor número de lechones nacidos vivos por camada o por parto, y tratar de mantenerlos desde la fase del destete hasta la de engorde. 
Fase de destete: en esta fase la cerda es retirada al área de reproducción hasta la nueva cubrición o apareamiento, quedando los lechones, normalmente, en el mismo sitio de lactancia durante aproximadamente una semana, iniciando un período independiente de la madre, es decir, deja de alimentarse con la leche materna y se comienza a hacer con el alimento balanceado Preiniciador; se hace un seguimiento al control sanitario, para luego pasar a la fase de levante, con un peso promedio de $15 \mathrm{kgs}$ por lechón. La mejor forma de conocer la conversión del kilogramo de alimento en kilogramo de carne es utilizando una balanza para pesar los cerdos al inicio y finalización de cada etapa, y saber así el peso promedio.

La balanza no sólo es importante para determinar el peso promedio de los cerdos en cada fase del proceso de producción, también permite medir el grado de conversión del alimento día por día en cada una de dichas fases, evaluar el sistema de alimentación, detectar cualquier inconveniente en el desarrollo del animal, comprobar el peso adecuado y la madurez sexual de las hembras de reemplazo y hasta el desgaste sufrido por la reproductora durante la lactancia.

Fase de levante: en esta fase los cerdos permanecen aproximadamente sesenta (60) días, utilizando el iniciador como tipo de alimento, con dos raciones diarias, una en la mañana y otra en la tarde, con un promedio de 2 kgs por día. En esta etapa se deben separar por sexo para escoger a los nuevos reproductores de ambos sexos, si se decide por esta opción; los machos restantes se deberán castrar, es decir, extirpar los órganos genitales, además, de bañar diariamente, realizar la limpieza y desinfec- ción de los corrales, tomando en cuenta, además, la densidad animal, es decir, la superficie por cerdo y las condiciones ambientales, como la temperatura, la ventilación, entre otros. Salen con un peso promedio de 40 kgs.

Fase de crecimiento y desarrollo: en esta fase los cerdos permanecen un promedio de 30 días, utilizando el tipo de alimento denominado Desarrollo. El consumo de alimento es más alto, debido al rápido crecimiento del mismo, además, el porcentaje de mortalidad es menor y salen con un peso promedio de $60 \mathrm{kgs}$.

Fase de engorde: la permanencia de los cerdos en esta etapa es de aproximadamente 45 días, utilizando el tipo de alimento denominado Terminador, y no es suministrado por ración sino a voluntad del animal, obteniendo un peso promedio de 100 kgs, de acuerdo también al potencial genético y a las prácticas sanitarias adecuadas (Diagrama 1).

El objetivo de toda granja porcina, además, de obtener el mayor número posible de lechones vivos por camada o por parto, es la calidad de la carne exigida por el mercado para el consumo humano, en el menor tiempo y al menor costo, por eso de acuerdo a Moreno (2000), esta relación se puede obtener en un período cinco meses y medio (5 1/2). Además, Guzmán (2007:13) establece "una conversión promedio de alimento de $3,5 \mathrm{kgs}$ por kilogramo de carne, lo que significa que se requiere, cerca de $350 \mathrm{kgs}$ de alimento por cerdo para lograr una buena rentabilidad". De allí que, no obstante, a pesar de los controles regulatorios de los gobiernos de turno en Venezuela, sobre los precios de venta al público de la carne de cerdo, todavía esta actividad resulta ser muy 
Costos de producción en explotaciones porcinas de ciclo completo en el Municipio Mara... Rodríguez Medina, Guillermo; Rodríguez Castro, Belkis y Villasmil, Amaloa Karina

\section{Diagrama 1}

Proceso de producción cría de cerdos

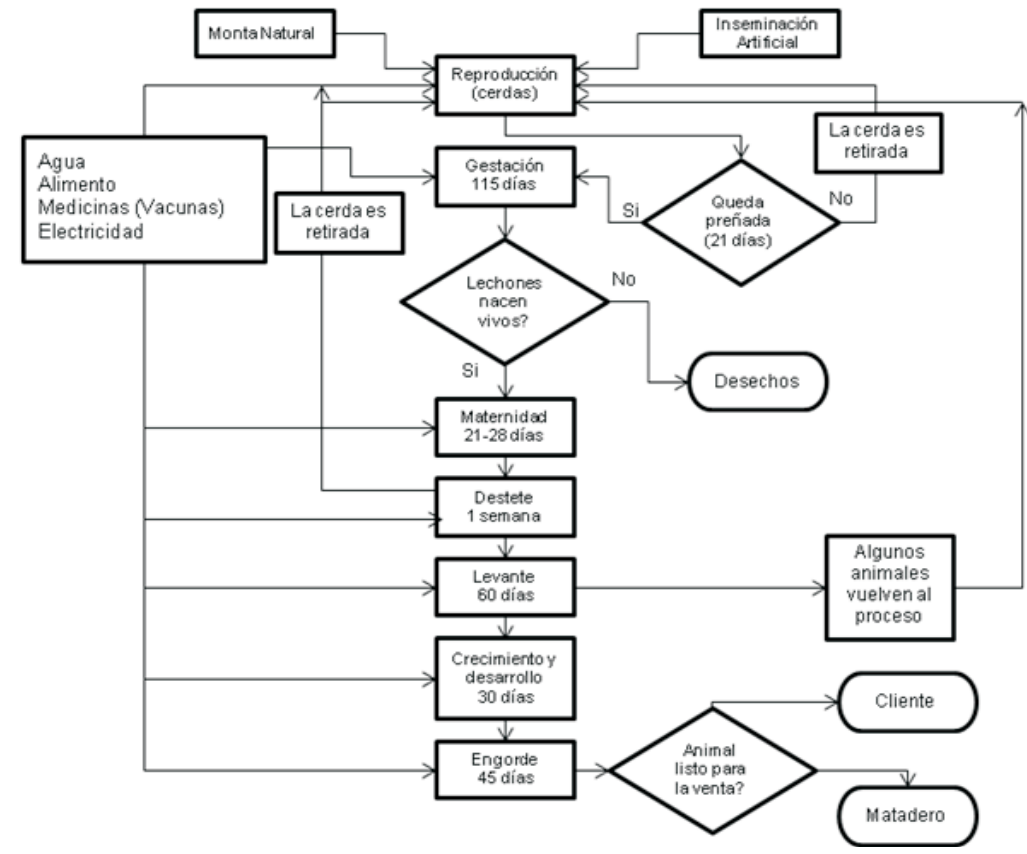

Fuente: Elaboración propia (2012).

redituable siendo bien gerenciada, desde el punto de vista de los costos, reproducción, genética, nutrición, sanidad y manejo, entre otros.

Con la fase de engorde se termina el proceso de producción en las granjas porcinas de ciclo completo o cerrado y el animal se encuentra listo para la venta o para su pase al matadero, bien sea para el consumo humano o para seguir con el procesamiento agroindustrial.

Al tener claro el productor de cerdos las diferentes fases que se desarrollan en el proceso de producción, debe llevar una planificación y un control que le permita determinar el costo de producción y saber su índice de rentabilidad, a través de los regis- tros, por lo menos de manera extracontable, utilizando formatos para tal fin.

\subsection{Producción porcina en uno o varios sitios}

La producción porcina tradicional consiste en que todas las fases del proceso de producción: reproducción, gestación, maternidad o lactancia, destete, levante, desarrollo y crecimiento, y engorde, se llevan en un mismo lugar, es decir, "todas las fases de producción se encuentran en los terrenos de la propia granja o sus alrededores, ya sea en un edificio o en varios edificios separados entre sí por 10 a 20 yardas" (Harris, 2001:39); en metros de 9 a 18. 
La producción en un solo sitio, normalmente es continua, lo cual significa que las salas de las diferentes fases siempre contienen animales de alguna edad, sin embargo, algunos productores consideran mejor al flujo de cerdos todo adentro y todo afuera, debido a que todos los cerdos de una camada (adentro) salen de la granja (afuera) para evitar que algunos agentes infecciosos puedan generar casos de neumonías u otras enfermedades si se mezclan con los de otros partos.

Por otro lado, se tiene la producción Isowean multisitio para evitar vaciar la granja por completo motivado a la aparición de alguna enfermedad. Esta producción consiste en que las fases del proceso de producción se llevan a cabo en granjas diferentes, y pueden ser dos o tres sitios, con la finalidad de evitar agentes infectocontagiosos. De esta manera se producen cerdos con mayor nivel sanitario, se aprovecha mejor el rendimiento, en cuanto a la calidad de la carne, a una mayor tasa de crecimiento y al índice de conversión del alimento, principalmente en la fase de engorde, además, de hacer uso de la economía de escala para tener un menor costo de producción, en comparación con los costos que se generan en las granjas de un solo sitio.

La palabra Isowean, de acuerdo a Harris (2001:43) "procede de Isolated Weaning (destete aislado), término que describe perfectamente la idea de separar a los lechones lactantes en el momento del destete de cualquier otro cerdo, sea cual sea su edad". De allí que la intención originalmente era para eliminar los agentes infecto-contagiosos presentes en la fase de reproducción, gestación y maternidad, y de esta manera pasarlos para otros sitios en las diferentes fases posteriores, hasta llegar a la de engorde con un mayor nivel sanitario y mejor aprovechamiento del rendimiento.

\subsection{Importancia de la inseminación artificial}

La inseminación artificial (IA) es una técnica de reproducción que se ha incrementado ampliamente en los últimos años, para el mejoramiento de la producción, y consiste en la extracción del semen del verraco, sometido a un proceso riguroso de conservación en condiciones óptimas, para ser depositado en el aparato genital de la hembra por medio instrumental.

La IA posee una serie de ventajas, ante la monta natural, que de acuerdo a Sábata (2008), Hafez, E.S.E. y Hafez (2002), Serie Agronegocios (2001), Daza (S/A) y Buxadé et al (1996), son las siguientes:

Ventajas para disminuir costos: Con la IA se tiene menos verracos y esto hace que a) haya menor consumo de alimentos, b) se necesita menos corrales, c) mejor aprovechamiento del espacio, d) mejor empleo y menos costo en la mano de obra, porque se necesita menos personal, e) se obtiene una mayor ganancia de peso, al tener pocos verracos con un alto valor genético y mayor índice de conversión, f) se ahorra tiempo en la inseminación artificial, por lo tanto también se ahorra costo, pues, ella se hace aproximadamente en cinco minutos, g) con un verraco, según Allende y Omaña, entrevista (2011), se puede servir hasta 200 hembras por mes, mientras que por monta natural la relación es de uno por veinte 
Costos de producción en explotaciones porcinas de ciclo completo en el Municipio Mara... Rodríguez Medina, Guillermo; Rodríguez Castro, Belkis y Villasmil, Amaloa Karina

(20) cerdas como máximo; además, Artur et al. (1991:636), indican que "se pueden realizar aproximadamente 30 inseminaciones con cada eyaculación y se necesita una gran cantidad de semen diluido para cada inseminación (50 a $500 \mathrm{ml}$ )".

Ventajas de manejo: a) hay ahorro de tiempo, manejo y esfuerzo al evitar la monta natural y el desplazamiento de los reproductores, sean verracos o hembras para realizar la cubrición o apareamiento, b) se eliminan las lesiones y traumatismos en los verracos o de las cerdas, que puedan ocurrir al momento de realizar los servicios, c) se pueden usar verracos pesados de gran valor genético para servir a hembras jóvenes y d) se evita el stress de animales con problemas cardíacos o de abstención durante la monta.

Ventajas sanitarias: a) se reduce el riesgo de transmisión de enfermedades infecto-contagiosas por vía sexual, b) permite controlar la calidad espermática de los sementales que están sujetos a múltiples efectos medioambientales y sanitarios, c) se reduce la entrada de animales portadores de enfermedades del exterior y d) se mejora el estatus sanitario de la granja.

Ventajas zootécnicas: Con la disminución del número de verracos, hay ahorro de espacio, costos de mantenimiento y se obtiene un buen programa de mejoramiento de rendimientos, utilizando el semen de verracos de alto valor genético para su difusión y mejora de la reproducción.

Todo esto indica que al utilizar la IA se obtiene grandes ventajas no solo desde el punto de vista de la reducción de los costos, sino también de otra naturaleza, como se indicó anteriormente, y hace que se incremente la calidad de la carne y se aumente la productividad. Todo esto concuerda con lo señalado por Fuentes (2007:51), cuando indica que, "sin duda alguna, en la actualidad, la herramienta reproductiva más eficaz en el mejoramiento de la productividad en las empresas (granjas) porcinas es la inseminación artificial".

\section{Costos de producción: consideraciones teóricas}

Antes de dilucidar sobre los costos de producción es necesario contextualizar el término costo, el cual de acuerdo a Hansen y Mowen (2007:35) es el efectivo o un valor equivalente de efectivo sacrificado por productos y servicios que se espera que aporten un beneficio presente o futuro a una organización; para Blocher et al. (2008:55) se lleva a cabo un costo cuando un recurso es utilizado para cierto propósito, Del Río (2003: II-9), manifiesta que es la suma de esfuerzo y recursos que se han invertido para producir algo.

El costo no es más que el recurso escaso sacrificado para producir o adquirir bienes tangibles o intangibles para generar beneficios presentes o futuros y cuando esto sucede el costo expira y se convierte en gasto.

Los costos de producción indudablemente es la cuantificación de los diferentes recursos utilizados en dicho proceso y deben gerenciarse adecuadamente para potenciar el éxito de las explotaciones porcinas, a través de la planificación y el control, la búsqueda de la eficiencia para incrementar la productividad y poder alcanzar los objetivos planteados, tal 
como lo señala Laurentin (2000:11), cuando indica que,

"La administración estratégica es el proceso a través del cual las organizaciones (granjas) previo un diagnóstico del medio y análisis de sus condiciones internas, determinan en términos generales (estrategias) y especificas (actividades), su dirección futura, así como también implantan las decisiones tendientes a lograr sus objetivos a largo y corto plazo".

El costo de producción de acuerdo a Rodríguez et al. (2007:459) es "el conjunto de costos, compuesto por la adquisición de los materiales directos e insumos, y el esfuerzo que se incurre por la realización de los procesos y actividades para la obtención de un bien tangible o intangible", es decir, está referido al costo de la materia prima y de los insumos, al de la mano de obra directa y a los costos indirectos de producción, los cuales deben ser recolectados, medidos y acumulados de una manera sistemática para determinar los costos de producción.

El interés fundamental de la contabilidad de costos es rastrear el costo de los elementos que intervienen en la producción o servucción de un bien tangible o intangible en una explotación, mientras que la gestión de costos se refiere a las estrategias y acciones que se llevan a cabo de una manera sistematizada, a través de la planeación y el control de las diferentes actividades generadoras de costos para potenciar el éxito gerencial de una organización.

Las explotaciones porcinas deben realizar una buena gestión de costos de producción en aras de buscar la eficiencia de los recursos utilizados e incrementar la productividad, de allí que, Gabosi (2011:18) indica que algunos de los principales argumentos para determinar los costos son:

"Conocer los márgenes que resultaran de la operación comercial;

- comparar los costos resultantes con los costos presupuestados y corregir los desvíos;

- conocer los costos con precisión permite compararse con la competencia y determinar las fortalezas y/o debilidades de cada uno;

- tomar decisiones de inversión o cambios con objetivos mensurables;

- proyectar mejoras operativas cuantificando el impacto de los mismos".

\subsection{Costos directos e indirectos de producción}

Los costos directos son aquellos que están relacionados directamente con el costo de producción de las granjas porcinas y se le puede hacer un seguimiento económicamente factible, es decir, de fácil identificación, tal como lo indica Blocher et al (2008:55), en el sentido que, "un costo directo puede rastrearse directamente de manera económica y conveniente a un grupo de costos o un objeto de costo".

Los costos directos son conocidos también como costos primos, los cuales están compuestos por los materiales y suministros directos y la mano de obra directa. Los costos directos de producción en las granjas porcinas están compuesto por los alimentos (que incluye también los fletes e impuestos), los medicamentos, las vacunas, el material de inseminación 
Costos de producción en explotaciones porcinas de ciclo completo en el Municipio Mara... Rodríguez Medina, Guillermo; Rodríguez Castro, Belkis y Villasmil, Amaloa Karina

artificial, la mano de obra directa de los trabajadores operativos (incluyen todos los beneficios adicionales), honorarios de médicos veterinarios, entre otros, es decir, lo que están directamente relacionados con el proceso de producción.

Dentro de los costos directos el de mayor significación es el alimento, principalmente el balanceado, ya que dentro de la estructura de costo representa aproximadamente al $80 \%$, y los productores del municipio estudiado lo compran a empresas dedicadas a esta labor, para las diferentes fases del proceso de producción (preiniciador, iniciador, desarrollo y terminador), por lo que es necesario un programa de alimentación.

Siendo el alimento, el recurso de mayor peso dentro de la estructura de costo, sería conveniente que los productores se agruparan para constituir por lo menos una cooperativa que elabore su propio alimento balanceado, con la finalidad de obtener un importante ahorro en los costos de producción.

Los costos indirectos son aquellos que no están relacionados directamente con el proceso de producción en las granjas porcinas y se dificulta hacerles un seguimiento para cargárselo al cerdo, ya que no son de fácil identificación en cada fase del proceso de producción, pero son necesarios para llevar a cabo de forma eficaz dicho proceso; estos son adjudicados a través de un método asignación de costos. En tal sentido, Horngren et al. (2007:27), señalan que, "los costos indirectos de un objeto del costo se relacionan con un objeto de costo en particular, pero no pueden rastrearse a ese objeto de manera económicamente factible (efectiva en cuanto a costos se refiere)".
Los costos indirectos de producción en las granjas porcinas lo componen: depreciaciones de las instalaciones y de maquinarias, seguros e impuestos, servicios públicos, combustibles, productos de limpieza, supervisión, alquileres, vestuarios y calzados del personal, almacén, entre otros.

\subsection{Proceso, actividades y recursos}

Para determinar el costo de producción de las explotaciones porcinas es necesario conocer el o los procesos que lo componen, de allí que Lorino (1993: 36), señala que, "se llamarían procesos al conjunto de actividades destinados a la consecución de un objetivo global, a una salida global, tanto material como inmaterial", mientras que Álvarez et al. (1996: 307), lo definen, “... como un conjunto coordinado de tareas que desembocan en la obtención de un producto o de un servicio especifico".

Basado en las apreciaciones anteriores, se tiene que los procesos de las granjas porcinas están integrados por las diferentes fases, que operan bajo unos procedimientos coordinados en forma sistemática, que trascienden el objetivo funcional de la fase con el norte de alcanzar el global de la explotación. Esta situación no es tomada en cuenta por los productores del municipio Mara, pues no tienen la mínima formación gerencial para llevar a cabo esta concepción de procesos, solo saben algunas de las fases del proceso de producción antes descritas sin el conocimiento detallado de las actividades que ellas encierran.

En cuanto a las actividades, de acuerdo a Sáez et al. (1993:187), "una actividad es un conjunto de tareas o actos imputables a un grupo de personas o a 
una persona, a un grupo de máquinas o a una máquina, y relacionadas con un ámbito preciso de la empresa", mientras que para Brimson (1997:26), "una actividad describe lo que la empresa hace, la forma en que el tiempo se consume y las salidas (outputs) de los procesos".

Las actividades están referidas al conjunto de tareas realizadas por una o varias personas en cada fase del proceso de producción, que partiendo del requerimiento y el consumo de una serie de recursos se obtiene un resultado determinado, es decir, es todo aquello que los trabajadores de la granja realizan día a día, hora tras hora para obtener un fin. Todo esta situación permite determinar con mayor exactitud el costo de producción en las explotaciones porcinas, para lo cual es necesario, de acuerdo a Rodríguez et al. (2009:264), desagregar dicho termino, "en aspectos como la identificación de ellas, el diccionario, el alcance, la unidad de análisis, la clasificación, los atributos, la capacidad, la jerarquía, los inductores y el sistema de información".

En este caso los productores no tienen definido como tal las actividades que intervienen en cada uno de los procesos, sino que los pocos trabajadores que poseen realizan sus tareas de acuerdo a los requerimientos de la granja, sin tener especificada sus labores dentro de las actividades que se encuentran en el o los procesos, por lo que sus costos no se distribuyen entre las diferentes actividades que realizan.

En relación con los recursos, están referidos a los diferentes elementos que son requeridos y consumidos (alimento, insumos de sanidad e higiene, gastos de personal, servicios públicos, uso de las maquinarias, entre otros) por las activi- dades que se realizan en el proceso de producción de las granjas porcinas, y se tiene que cuantificar su costo. En este sentido los costos de los recursos son imputados a las actividades y el costo de éstas es asignado a los cerdos, pero en el caso de las granjas objeto de estudio, no asumen este enfoque pues, son pocos productores que llevan registros $y$, los que los llevan lo hacen en un cuaderno donde se registran solamente los costos de los alimentos y de sus operarios (sin considerar sus beneficios laborales), no toman en cuenta los demás recursos utilizados, para determinar a grosso modo sus costos y rentabilidad. Por tales razones se considera que este trabajo será de ayuda a los productores para que realicen una gestión más adecuada de sus costos de producción.

\subsection{Sistema de costeo}

Existen diferentes sistemas de costeo, como el de ordenes de trabajo, por procesos, híbridos y basado en actividades, pero dado las características de las explotaciones porcinas, el sistema de costeo más adecuado es el costeo por operaciones, que es un costeo hibrido, el cual es una combinación del de ordenes de trabajo y por proceso, donde la acumulación de los costos de las diferentes fases del proceso de producción se llevarían a través del costeo por proceso y cada camada o lotes con características particulares se haría por el costeo de ordenes de trabajo, tal como lo señalan Hansen y Mowen (2007:249), cuando indican que, "los procesos de producción por lotes elaboran lotes de diferentes artí- 
Costos de producción en explotaciones porcinas de ciclo completo en el Municipio Mara... Rodríguez Medina, Guillermo; Rodríguez Castro, Belkis y Villasmil, Amaloa Karina

culos que son idénticos en muchos aspectos pero diferentes en otros".

En los actuales momentos los granjeros porcinos del municipio Mara no llevan ningún sistema de costeo que les permita determinar su costo de producción, pues, ni siquiera el de la contabilidad financiera, por lo tanto hay que incentivarlos a realizar este tipo de control.

\section{Costos de producción en explotaciones porcinas del municipio Mara}

Después de haber abordado el marco conceptual sobre el cual se sustentó el trabajo de investigación, en esta sección se analizan los resultados obtenidos, de las entrevistas a los productores, a través del cuestionario, para confrontar la realidad empírica con el marco de referencia.

De las diecinueve (19) granjas de ciclo completo, se encontraron quince (15) que tienen entre nueve (9) y veinte (20) cerdas para la reproducción, dos (2) entre veintiuno (21) y cincuenta (50) y dos (2) con más de cincuenta; en cuanto a los machos, se consiguió que dieciséis (16) granjas tienen entre uno (1) y dos (2) verracos, dos (2) explotaciones tienen tres (3) y una tiene cinco (5). Esta relación concuerda con lo manifestado por Allende y Omaña, Entrevista (2011), en el sentido que un verraco puede servir a veinte (20) cerdas como máximo.

En cuanto a la pregunta si se realiza la inseminación artificial (IA), solamente una respondió afirmativamente, es decir, 5,3\%, pues, esa granja posee doscientos ochenta y ocho (288) reproductoras, lo que significa que este productor sabe a ciencia cierta las ventajas de la IA en cuanto a la reducción de costos, en el manejo, en el aspecto sanitario y en las zootécnicas, tal como lo contempla Sábata (2008), Hafez y Hafez (2002), Serie agronegocios (2001), Daza (S/A) y Buxade et al. (1996).

En relación a si existe un área destinada a cada fase del proceso de producción, se encontró que $15,79 \%$, representado por tres (3) granjas tienen determinada la superficie destinada para cada una de dichas fases, mientras que $84,21 \%$, considera como una sola fase desde la de levante hasta el engorde. Estando este último porcentaje en contraposición con lo indicado por el Centro Nacional de producción más limpia de Honduras (2009), Sábata (2008) y Moreno (2000). Esto indica que se debe concientizar concientizar a los productores sobre la importancia de tener bien claro cada fase del proceso de producción, en aras de mejorar la eficiencia de los recursos y la obtención de un mejor rendimiento en sus explotaciones.

En la fase de parto y maternidad, fase crítica en el proceso, se desarrollan tres momentos: el de preparatoria, una semana antes del parto, el de la expulsión de los fetos y el de la placenta, la cual amerita en ambas una atención especial. Los resultados indican que el $73.7 \%$ (14 granjas) manejan una fecha probable de parto, además de tener un programa de asistencia al momento del mismo, tal como lo indica Daza (1995) y Whittemore (1998) citados por Sábata (2008), con la finalidad de que el parto transcurra de manera normal y facilite el mayor número de lechones nacidos vivos; se observó que 26,30\%, o sea, cinco granjas estipulan un tiempo promedio entre 21 y 28 , mientras que $73,7 \%$ indicó un tiempo superior. 
Referente al tiempo promedio de duración de la cerda en cada fase del proceso de producción, se encontró que en la etapa de reproducción $52,63 \%$ (10 granjas) consideran dos (2) o tres (3) días para la cubrición o apareamiento, lo cual concuerda con lo estipulado por el Centro Nacional de producción más limpia de Honduras (2009), Sábata (2008) y Moreno (2000); mientras que los otros productores esperan hasta una semana; en la fase de gestación, $63,15 \%$ toma en cuenta los estándares establecidos de 114 a 115 días, de allí que esto se conoce dentro del argot de los productores como 3,3,3 que significa tres meses, tres semanas, tres días.

En la fase de destete, sólo 5,3\% (1 granja) manifestó un tiempo promedio de siete (7) días, en concordancia con los estándares establecidos, y 94,7\% considera un lapso mayor, lo que significa el poco control que se tiene sobre el manejo del tiempo para alcanzar un mejor rendimiento del animal.

Merece atención especial las interrogantes sobre las áreas destinadas a las fases de levante, desarrollo y crecimiento, y la de engorde, por cuanto $78,9 \%$, es decir, quince (15) productores consideran la misma área para estas fases, mientras que tres (3) las tienen bien identificadas, absteniéndose una en responder. Esta circunstancia no permite que los productores aprovechen al máximo la conversión en carne, pues, en cada una de ellas se utiliza un tipo de alimento diferente, además, es conveniente utilizar una balanza para el control de la conversión en cada fase.

Con respecto a si las diferentes fases del proceso de producción se concentran en uno o varios sitios, se observó que $100 \%$ concentran sus operaciones en un solo sitio (producción tradicional y continua), es decir, siempre hay cerdos de cualquiera edad en las diferentes fases del proceso. Pero también existen productores que su producción de cerdos es Isowen multisitios, donde las fases del proceso de producción lo llevan a cabo en granjas diferentes y distantes para evitar los agentes infectocontagiosos, que no es el caso en esta investigación.

En cuanto a la determinación del costo de producción, se consiguió que $5,3 \%$ (una granja) lleva un sistema contable manual, no de costo sino financiero, y su administradora asegura que le permite determinarlo, sin embargo, lo más conveniente es el establecimiento de un sistema de costo, tal como lo señalan Rodríguez et al. (2007); además, 21,1\% (cuatro (4) explotaciones) llevan unos cuadernos con anotaciones, mientras que $73,6 \%$ no presenta ningún tipo de control, para saber su costo de producción, pero dichos productores $(30 \%$ a $40 \%)$ manifestaron que por experiencia propia la actividad porcina es rentable, a pesar de los controles regulatorios establecidos por los entes gubernamentales.

Referente al conocimiento de las actividades que se involucran en el proceso de producción, se consiguió que $21,1 \%$, (cuatro (4) productores) respondieron en forma afirmativa, sin embargo, surgió una contradicción en cuanto a los granjeros que manifestaron conocer las fases del proceso de producción, las cuales fueron enunciadas en el cuestionario, (reproducción, gestación, parto y materidad, destete, levante, crecimiento y desarrollo y engorde), y tres (3) aseveraron saberlo, en otras palabras, $15,79 \%$. En este 
Costos de producción en explotaciones porcinas de ciclo completo en el Municipio Mara... Rodríguez Medina, Guillermo; Rodríguez Castro, Belkis y Villasmil, Amaloa Karina

sentido, se observó que un porcentaje muy bajo tiene conocimiento sobre los procesos y actividades.

En relación al ítem sobre los costos directos que integran los costos de producción en las diferentes fases del proceso, como son los de alimentos, sanidad e higiene, mano de obra directa, asesoramiento, entre otros, la cual se hizo cerrada, 100\% de los productores expresaron que el costo de alimento es el que tienen mayor peso dentro de ellos; sin embargo, sólo $52,63 \%$ (diez (10) granjeros) estimaron su ponderación entre $70 \%$ y $80 \%$, mientras que el resto no indicó nada sobre el particular. Siguiendo en orden de importancia los costos de mano de obra directa y los de sanidad e higiene, respectivamente, pero sin especificar su porcentaje.

Con respecto a los costos indirectos de producción: depreciaciones de las instalaciones y maquinarias, mantenimiento, servicios públicos, entre otros (pregunta cerrada en el instrumento diseñado), no informaron nada sobre los mismos, porque desconocen su cuantificación y distribución.

Por otro lado, ninguna de las explotaciones porcinas encuestadas en el mu- nicipio Mara elabora su propio alimento balanceado, siendo que $94,7 \%$ (dieciocho (18) de los diecinueve granjeros entrevistados) lo compran a proveedores, y solo uno $(5,3 \%)$ utiliza las vísceras y otros desperdicios agrícolas para su alimentación, sin embargo, existen varios productores que combinan el alimento balanceado con otros tipos de alimentos.

Referente al sistema de costeo utilizado para determinar sus costos de producción, ninguna de las explotaciones encuestadas usa contabilidad de costos, por tanto no lo aplican, como lo refiere Hansen y Mowen (2007), Horngren et al. (2007) y Rodríguez et al. (2007).

Ante la falta de control para determinar los costos de producción se les elaboró y entregó unos formatos a algunos productores para su distribución; estos facilitaran el uso racional de los recursos que utilizan, tanto en unidades físicas como monetarias, para llevar a cabo sus labores, y tener por lo menos de manera extracontable herramientas que le faciliten conocer y aumentar su eficiencia productiva (Cuadros del 1 al 5).

\section{Cuadro 1}

Control Individual por Cerda

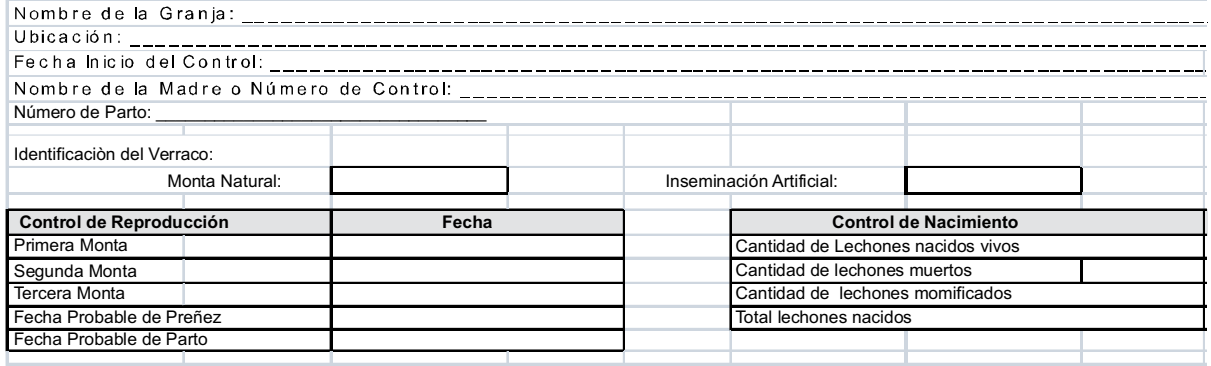

observaciones:

Fuente: Elaboración propia (2012). 
Cuadro 2

Control por Camada

\begin{tabular}{|c|c|c|c|c|c|c|c|c|c|c|c|c|c|c|c|c|}
\hline & & & & & & & & & & & & & \\
\hline \multicolumn{2}{|c|}{ Ubicación: } & & & & & & & & & 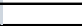 & & & & & & \\
\hline \multirow[b]{2}{*}{$\begin{array}{l}\text { \# de } \\
\text { Lechones } \\
\text { nacidos } \\
\text { vivos }\end{array}$} & \multirow[b]{2}{*}{$\begin{array}{l}\text { Fecha } \\
\text { de } \\
\text { Naci- } \\
\text { miento }\end{array}$} & \multicolumn{2}{|c|}{ Sexo } & \multirow[b]{2}{*}{$\begin{array}{c}\text { Prome- } \\
\text { dio de } \\
\text { peso } \\
\text { al } \\
\text { nacer }\end{array}$} & \multicolumn{4}{|c|}{ Destete } & \multicolumn{3}{|c|}{ Desarrollo y } & \multicolumn{3}{|c|}{ Engorde } & \multicolumn{2}{|c|}{ Venta } \\
\hline & & M & H & & \begin{tabular}{|c|} 
No. \\
Lechones \\
Vivos
\end{tabular} & Bs & \begin{tabular}{|c|}
$\mathrm{Kg}$ \\
Alimen- \\
to con- \\
sumido
\end{tabular} & \begin{tabular}{|l|} 
Peso \\
Prome- \\
dio de \\
la fase
\end{tabular} & $\begin{array}{l}\text { No. } \\
\text { Cerdos } \\
\text { Vivos }\end{array}$ & \begin{tabular}{|c|}
$\mathbf{K g}$ \\
Alimento \\
Consu- \\
mido
\end{tabular} & $\begin{array}{c}\text { Peso } \\
\text { Pro- } \\
\text { medio } \\
\text { de la } \\
\text { fase }\end{array}$ & $\begin{array}{c}\text { No. } \\
\text { Cer- } \\
\text { dos } \\
\text { Vivos }\end{array}$ & $\begin{array}{c}\mathrm{Kg} \text { Ali- } \\
\text { mento } \\
\text { Consu- } \\
\text { mido }\end{array}$ & $\begin{array}{c}\text { Peso } \\
\text { Pro- } \\
\text { medio } \\
\text { de la } \\
\text { fase }\end{array}$ & $\begin{array}{l}\text { Fecha } \\
\text { de } \\
\text { Venta }\end{array}$ & Bs \\
\hline & & & & & & & & & & & & & & & & \\
\hline & & & & & & & & & & & & & & & & \\
\hline & & & & & & & & & & & & & & & & \\
\hline & & & & & & & & & & & & & & & & \\
\hline & & & & & & & & & & & & & & & & \\
\hline & & & & & & & & & & & & & & & & \\
\hline O & & & & & & & & & & & & & & & & \\
\hline Observaci & ones: & & & & & & & & & & & & & & & \\
\hline
\end{tabular}

Fuente: Elaboración propia (2012).

Cuadro 3

Control de Existencia de Alimentos

\begin{tabular}{|c|c|c|c|c|c|c|c|c|c|c|c|c|c|}
\hline \multicolumn{14}{|c|}{ Nombre de la Granja: } \\
\hline \multicolumn{14}{|c|}{ Ubicación: } \\
\hline \multicolumn{14}{|c|}{ Alimento: } \\
\hline & & & & & \multirow{2}{*}{\multicolumn{3}{|c|}{ Entradas }} & \multirow{2}{*}{\multicolumn{3}{|c|}{ Salidas }} & & & \\
\hline & & \multicolumn{3}{|c|}{ Inventario Inicial } & & & & & & & \multicolumn{3}{|c|}{ Inventario Final } \\
\hline Fecha & Detalle & Cantidad & $\begin{array}{c}\text { Costo } \\
\text { Unitario }\end{array}$ & BsF & Cantidad & $\begin{array}{l}\text { Costo } \\
\text { Unitario }\end{array}$ & BsF & Cantidad & $\begin{array}{l}\text { Costo } \\
\text { Unitario }\end{array}$ & BsF & Cantidad & $\begin{array}{l}\text { Costo } \\
\text { Unitario }\end{array}$ & BsF \\
\hline & & & & & & & & & & & & & \\
\hline & & & & & & & & & & & & & \\
\hline & & & & & & & & & & & & & \\
\hline & & & & & & & & & & & & & \\
\hline & & & & & & & & & & & & & \\
\hline & & & & & & & & & & & & & \\
\hline & & & & & & & & & & & & & \\
\hline
\end{tabular}

Fuente: Elaboración propia (2012).

Cuadro 4

Control de Vacunación

\begin{tabular}{|c|c|c|c|c|c|c|c|}
\hline \multicolumn{8}{|l|}{ Nombre de la Granja: } \\
\hline \multicolumn{8}{|l|}{ Ubicación: } \\
\hline & & & & & & & \\
\hline \multirow{2}{*}{ Nombre de la Vacuna } & \multicolumn{6}{|c|}{ Fechas } & \multirow{2}{*}{ OBS } \\
\hline & 1era Dosis & 2da Dosis & 3era Dosis & Refuerzo & Refuerzo & Refuerzo & \\
\hline & & & & & & & \\
\hline & & & & & & & \\
\hline
\end{tabular}

Fuente: Elaboración propia (2012). 
Costos de producción en explotaciones porcinas de ciclo completo en el Municipio Mara... Rodríguez Medina, Guillermo; Rodríguez Castro, Belkis y Villasmil, Amaloa Karina

\section{Cuadro 5}

\section{Control General de los cerdos en la Granja}

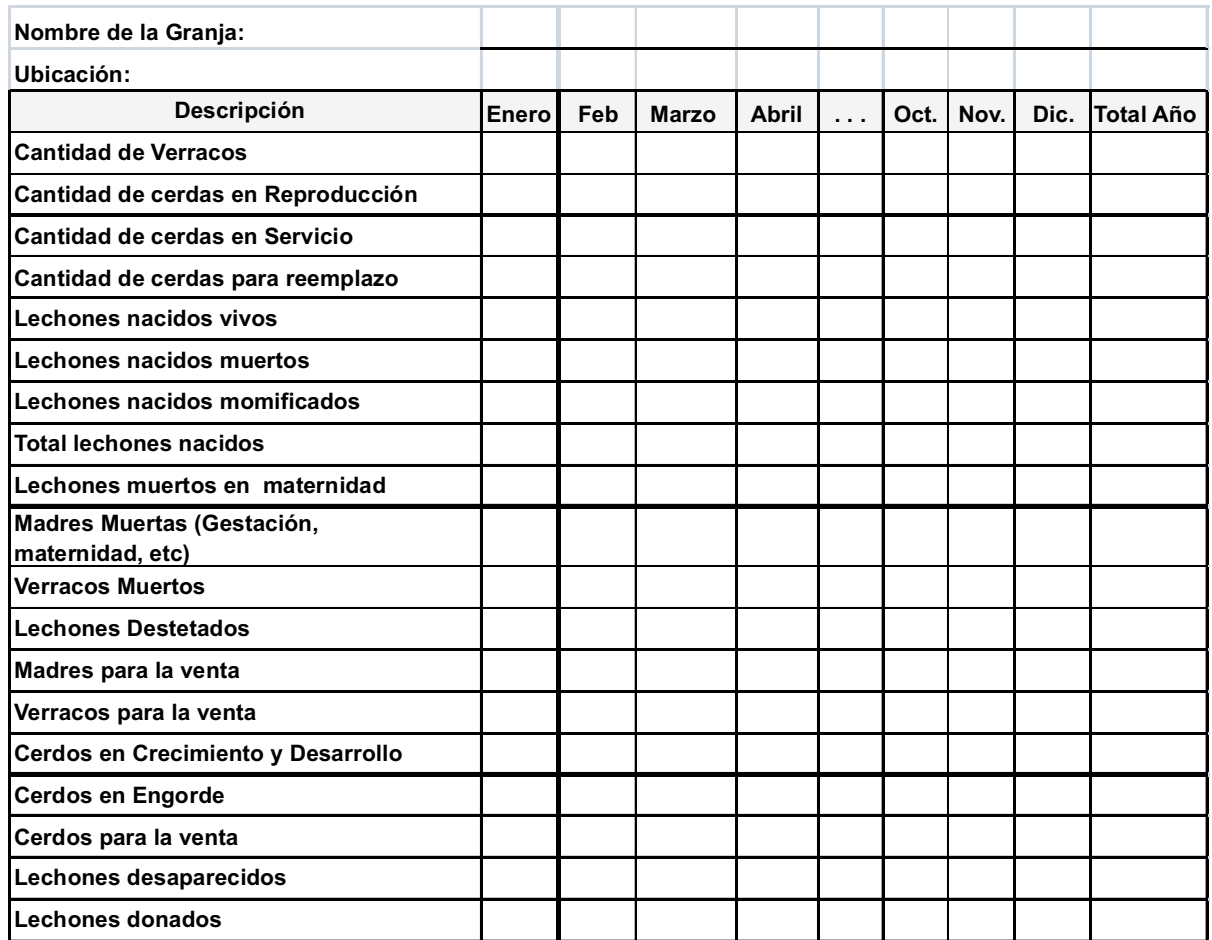

Fuente: Elaboración propia (2012).

\section{Conclusiones}

Los productores que se dedican a las explotaciones porcinas, objeto de estudio de esta investigación, tienen bien claro la redituabilidad de dicha actividad, no obstante los controles regulatorios gubernamentales en dicho sector, en cuanto al precio de venta al público de la carne para su consumo.

De acuerdo a los resultados se tiene que solamente existe en el municipio Mara una sola granja con doscientos ochenta y ocho cerdas reproductoras y es la única que aplica la inseminación artificial, aprovechando las grandes ventajas que ésta ofrece, en cuanto a la reducción del costo de producción, a mejorar el valor genético en beneficio de la reproducción, a la mejoras sanitarias y a las de manejo; mientras que el mayor número de granjas de ciclo completo tienen entre nueve (9) y veinte (20) reproductoras.

Los granjeros no le sacan el mejor provecho a sus explotaciones, debido a que desconocen las diferentes fases en que se desagrega el proceso de producción, solamente se hace mayor énfasis en 
las fases que van desde la reproducción a la de destete, ya que la de levante hasta la de engorde generalmente se considera como si fuera una sola; por otro lado, muy poco toman en cuenta el tiempo promedio de permanencia de los cerdos en cada una de ellas, en la búsqueda de la eficiencia de los recursos, la mejor conversión del alimento en carne y el mayor rendimiento de la explotación, pues, utilizan un tiempo de estancia superior a los estándares establecidos, ya que de acuerdo a estos, en cinco meses y medio $\left(5 \frac{1}{2}\right)$ se pueden obtener fácilmente cerdos con $100 \mathrm{kgs}$. de peso, ya que ahorrar tiempo es ahorrar costo y por ende obtener mayor rentabilidad.

Todas las granjas objeto de estudio utilizan un solo sitio de carácter continuo para la explotación de su actividad, es decir, siempre hay animales de alguna edad en las diferentes fases, y en ningún caso se consiguió la producción Isowen multisitio. Asimismo, no se determina el costo de producción y ninguna lleva un verdadero control de ellos, para poder cuantificar su rentabilidad, no obstante contar con elementos de juicio que les permite llevar sus costos en cada fase del proceso de producción, pues, los productores conocen sus costos directos, no así los indirectos, lo que les facilita la determinación de los costos por camada, y para ello se debe utilizar el costeo por lote u operaciones.

Este trabajo permite aseverar que solamente tres (3) productores conocen todas las fases de producción (reproducción, gestación, parto y maternidad, destete, levante, crecimiento y desarrollo y engorde) y solamente una lleva un sistema contable, que no es de costos sino financiero, que le permite determinar su costo de producción, mientras que los otros granjeros consideran las fases de levante, creación y desarrollo, y de engorde como una sola. Toda esta situación permite afirmar que los productores de cerdos del municipio Mara no determinan sus costos de producción, pero la experiencia les indica que dicha actividad económica es redituable.

Por otro lado, se tiene que este sector está desatendido tanto en su organización, para su desarrollo, como en la parte financiera, pues, ellos manifiestan su buena voluntad para mejorar sus procesos; pero dichas limitaciones les impide ser más eficientes y productivos, por lo que se recomienda que las entidades agropecuarias de los gobiernos municipales, regionales y nacionales deben hacerle frente a esta situación. También, se recomienda crear una cooperativa que les permita la elaboración de su propio alimento balanceado y venderlos a precios solidarios a los demás productores, con la finalidad de reducir sus costos producción y favorecer la soberanía y seguridad alimentaria.

\section{Referencias bibliográficas}

Álvarez, López; Amat Salas, Joan; Amat Salas, Oriol y Colaboradores (1996). Contabilidad de gestión Avanzada. Planificación, Control y Experiencias Prácticas. Mc Graw Hill. España.

Amarú, Antonio (2009). Fundamentos de administración. Teoría general y proceso administrativo. Editorial Pearson educación. México.

Araque, Humberto (2009). Sistema de producción de cerdos. Universidad central de Venezuela. Campus Maracay, Facultad de Agronomía. Instituto y Departamento de producción animal. Venezuela. 
Costos de producción en explotaciones porcinas de ciclo completo en el Municipio Mara...

Rodríguez Medina, Guillermo; Rodríguez Castro, Belkis y Villasmil, Amaloa Karina

Arthur, Geofrey; Noakes, David y Pearson, Harold (1991). Reproducción y obstetricia en veterinaria (Teriogenologia). Sexta edición. Interamericana. Mc Graw Hill. España.

Blocher, Edward; Stout, David; Cokin, Gary y Chen Kung (2008). Administración de costos. Un enfoque estratégico. Cuarta edición. Mc Graw Hill. México.

Brimson, James (1997). Contabilidad por actividades. Alfaomega grupo editor. México.

Buxadé Carbó, Carlos y Colaboradores (1996). Porcicultura intensiva y extensiva. Ediciones mundi-prensa. España.

Centro Nacional de Producción más limpia de honduras (CNP-LH). 2009. Guía de buenas prácticas ambientales para la producción porcina. Edición AGA \& Asociados - consultores en comunicación. Honduras.

Da Silva, Reinaldo (2003). Teoría de la administración. Editorial Thomson. México.

Daza Castañeda, Néstor (S/A). Manual básico de porcicultura. Asociación Colombiana de porcicultores. Fondo Nacional de a Porcicultura. Editorial Scripto Ltda. Colombia.

Del Río González (2003). Costos I. Vigésima primera edición. Editorial Thomson. México.

Fuentes, Armando (2007). Manipulación del semen porcino para ser utilizado en inseminación artificial. Articulo en Cuaderno científico Girarz 4. Reproducción e inseminación artificial porcina. Editor Armando Quintero. Ediciones Astro Data. Venezuela.

Gabosi, Horacio (2011). Gestión y control de los costos de alimentación porcina. Revista porcinews. Edición VLI. Venezuela.
Gadd, John (2005). Guía John Gadd de soluciones en producción porcina. Editorial Servet. España.

Guzmán, José (2007). El cerdo de carne. Zootecnia. Biología y explotación comercial. Segunda edición. Espasande SRL editores. Venezuela.

Hafez, E.S.E. y Hafez, B. (2002). Reproducción e inseminación artificial en animales. Séptima edición. Mc Graw Hill Interamericana. México.

Hansen, Don y Mowen, Maryanne (2007). Administración de costos. Contabilidad y control. Quinta edición. Editorial thomson. México.

Harris, D.L. ('Hank"). (2001). Producción porcina multisitio. Editorial Acribia S.A. España.

Horngren, Charles; Datar, Srikant y Foster, George (2007). Contabilidad de costos. Un enfoque gerencial. Duodécima edición. Pearson Prentice Hall. México.

Hurtado, Jacqueline (2010). Metolodogía de la investigación. Guía para la comprensión holística de la ciencia. Cuarta edición. Ediciones Quiron. Venezuela.

Laurentin R., Hernan (2000). Estrategia para el desarrollo sostenible de aéreas porcinas con baja incidencia de enfermedades. Revista Venezuela porcina. Año 14 No. 37.

Lorino, Philippe (1993). El control de gestión estratégico. La gestión por actividades. Ediciones Alfaomega. México.

Manuales para educación agropecuaria (2006). Porcinos. Área: producción animal 5. Editorial trillas. México.

Moreno Caldeon, Janadira (2000). Informe de prácticas profesionales (explotación de porcinos). Trabajo de grado para optar el titulo de Medica Veterinaria. Tutoreado por el Dr. Eulices Rodríguez y el Dr. Oswaldo Vale. Facul- 
tad de Veterinaria. Universidad del Zulia. Venezuela.

Parra, Javier (2006). Guía de muestreo. Tercera edición. Editado en la Facultad de Ciencia Económicas y Sociales de la Universidad del Zulia. Venezuela.

Parra, Javier (2006). Guía de muestreo. Tercera edición. Editado en la Facultad de Ciencias Económicas y Sociales de la Universidad del Zulia. Venezuela.

Rodríguez, Guillermo; Chávez, Jorge; Rodríguez, Belkis y Chirinos, Alira (2007). Gestión de costos de producción en el sector metalmecánico de la región zuliana. Revista de ciencias sociales. Volumen XIII. No. 3. Periodo septiembre-diciembre. Universidad del Zulia. Venezuela.

Rodríguez, Guillermo; Rodríguez, Belkis; Chirinos, Alira y Melean, Rosana (2009). Gestión de costos de las actividades en el sector metalmecánico de la región Zuliana. Revista venezolana de gerencia. Año 14 No. 46, Abril-Junio. Universidad del Zulia. Venezuela.

Sábata A., Anna (2008). La gestión de los costes en el sector ganadero porcino. Tesis doctoral Univeritat de Barcelona. Departament de comptabilitat. Dirigida por el Dr. D. Ferran Mir Estruch. Consultado en http://www.te- sisenxarxa.net/tesis ub/available/ tdx_0717108-123635//asa_tesis.pdf . Consultada el 20/11/11.

Sáez, Ángel; Fernández, Antonio y Gutiérrez, Gerardo (1993). Contabilidad de costes y contabilidad de gestión. Volumen I. Mc Graw Hill interamericana. España.

Serie Agronegocios (2001). Crianza de porcinos. Centro de estudios agropecuarios. Grupo editorial Iberoamericana. México.

Stoner James y Freman, Edward (1995). Administración. Quinta edición. Editorial Prentice hall Hispanoamericana. México.

Utrera Vitelio (2006). La producción de credos en Venezuela. Estamos listos para el Mercosur. Expoferia. www.feporcina.org/06noticias/exp/memorias/resumenes/sanidad\%20porcina/competitividaddelaproduccióndecerdosenvenezuela\%5B2\%5D.pdf

\section{Entrevistas}

Allende Palma, Miguel $\mathrm{H}$ y Omaña, Marianis M. (2011). Entrevista realizada en el Centro de Inseminación Artificial en la Granja El Nido, estado Zulia. Venezuela el 25-11-2011. 